\title{
Cerebellar rTMS in PSP: a Double-Blind Sham-Controlled Study Using Mobile Health Technology
}

\author{
Andrea Pilotto ${ }^{1,2}$ (D) Maria Cristina Rizzetti ${ }^{2}$ - Alberto Lombardi ${ }^{1}$ - Clint Hansen ${ }^{3} \cdot$ Michele Biggi $^{4}$. \\ Giacomo Verzeroli ${ }^{4}$. Antonella Martinelli ${ }^{4}$. Robbin Romijnders ${ }^{3}$ - Barbara Borroni ${ }^{1}$ - Walter Maetzler ${ }^{3}$. \\ Alessandro Padovani ${ }^{1}$
}

Accepted: 26 January 2021 / Published online: 5 February 2021

(C) The Author(s) 2021

\begin{abstract}
There are no effective treatments in progressive supranuclear palsy (PSP). The aim of this study was to test the efficacy of theta burst repetitive transcranial magnetic stimulation (rTMS) on postural instability in PSP. Twenty PSP patients underwent a session of sham or real cerebellar rTMS in a crossover design. Before and after stimulation, static balance was evaluated with instrumented (lower back accelerometer, Rehagait ${ }^{\circ}$, Hasomed, Germany) 30-s trials in semitandem and tandem positions. In tandem and semitandem tasks, active stimulation was associated with increase in time without falls (both $p=0.04$ ). In the same tasks, device-extracted parameters revealed significant improvement in area $(p=0.007)$, velocity $(p=0.005)$, acceleration and jerkiness of sway $(p=0.008)$ in real versus sham stimulation. Cerebellar rTMS showed a significant effect on stability in PSP patients, when assessed with mobile digital technology, in a double-blind design. These results should motivate larger and longer trials using non-invasive brain stimulation for PSP patients.
\end{abstract}

Keywords Progressive supranuclear palsy $\cdot$ Repetitive transcranial magnetic stimulation $\cdot$ Mobile health technology $\cdot$ Postural instability

\section{Introduction}

Progressive supranuclear palsy (PSP) is a neurodegenerative disorder characterised by akinetic rigid syndrome with ocular motor dysfunction, early postural instability and falls [1].

Despite potential limited benefit from dopaminergic drugs, there are still no effective treatments available for postural instability and falls. Recent imaging and neuropathology studies revealed a reduced volume of the cerebellum with Tau accumulation in PSP patients $[2,3]$. These evidences suggest that cerebellum may be a potential target for non-invasive stimulation, as already recently demonstrated for multiple sclerosis [4].

Andrea Pilotto

pilottoandreae@gmail.com

1 Neurology Unit, Department of Clinical and Experimental Sciences, University of Brescia, P.zale Spedali Civili, 1, 25123 Brescia, Italy

2 Parkinson's Disease Rehabilitation Unit, FERB Onlus Trescore Balneario, Bergamo, Italy

3 Department of Neurology, Christian-Albrechts-University of Kiel, Kiel, Germany

4 Physical Therapy Unit, Milano Bicocca University, Bergamo, Italy
Accordingly, neurophysiological studies demonstrated an impairment in functional connectivity between the cerebellar hemispheres and contralateral primary motor cortex (cerebellar brain inhibition, CBI) [5, 6]. A preliminary, open-label trial with 10 PSP patients showed an improvement of CBI using theta burst repetitive cerebellar transcranial magnetic stimulation (rTMS) [7]. Moreover, a case study showed improvement of posturography parameters secondary to cerebellar stimulation in two PSP patients [8].

Based on these promising results, we aimed at evaluating the effect of a single-session cerebellar rTMS in PSP patients. We applied a double-blind sham-controlled crossover design, including a standardised assessment of static balance using mobile health technology.

\section{Methods}

\section{Study Cohort}

Consecutive PSP patients were recruited and underwent a review of the medical history, a neurological examination including the PSP rating scale (PSPRS) [9] and a 
comprehensive cognitive and behavioural assessment [10]. Inclusion criteria were (1) clinical diagnosis of probable PSP according to current criteria [1], (2) the ability to stand alone without support and (3) the ability to walk at least $3 \mathrm{~m}$ without aid. Exclusion criteria were (1) dementia, (2) vestibular/ proprioceptive or sensory abnormalities and (3) any contraindication to perform brain stimulation.

All subjects gave written informed consent prior to participation. The local ethics committee approved the study (protocol 193/16), recorded as NCT04222218 in clinicaltrial.gov. The study was performed in accordance with the Declaration of Helsinki.

\section{Repetitive Transcranial Magnetic Stimulation}

Each patient received both rTMS and sham cerebellar singlesession stimulations in randomised order in two different sessions performed at the same time of the day, separated by at least 2 weeks. The patient and the examiner were blind to the type of rTMS delivered, applied by another experimenter. Repetitive cerebellar theta burst stimulation was performed by Duo-Mag XT100 (Deymed -Horonov, Czech Republic) according to the protocol described by Brusa and coauthors [7]. The coil was placed tangentially to the skull over the lateral cerebellum $1 \mathrm{~cm}$ inferior and $3 \mathrm{~cm}$ right to the inion. Three $50-\mathrm{Hz}$ pulses were repeated at a rate of $5 \mathrm{~Hz} ; 20$ trains of 10 bursts with 8 -s intervals for a total of 600 pulses and for total time of 240 seconds were applied [7]. The intensity of rTMS was set at the $80 \%$ of the resting motor threshold obtained in the left motor cortex for each subject. For sham simulation, a spacer was attached to the coil; the stimulation parameters, the coil position and the sound were identical to the active condition.

\section{Dynamic Mobility and Mobile Health Technology- Instrumented Static Balance Assessment}

All subjects underwent a clinical evaluation including the Tinetti test, the Short Physical Performance Battery (SPPB), the Timed Up and Go test and the Functional Reach test (FR) before and after stimulation [11]. Static balance was tested before and after each stimulation with four tasks of 30-s duration, respectively: tandem and semitandem stance with eyes closed and with eyes open (Supplementary Figure 1). Primary endpoint was changes in the time in this position without support. For mobile health technology secondary outcomes, an inertial sensor unit (IMU) with $100 \mathrm{~Hz}$ sampling frequency (Rehagait $\AA$, Hasomed GmbH, Magdeburg, Germany) was fixed at the level of the third lumbar spine segment close to the centre of mass [11]. Acceleration signals were processed and calculated as previously described [12]. The following sway parameters were extracted: area, mean velocity, mean acceleration (root mean square - RMS), jerk (indicating smoothness of compensatory movements) and mean frequency [13]. Mean velocity, RMS and jerk were calculated for both anteroposterior (AP) and medio-lateral (ML) directions.

\section{Statistical Analyses}

Differences in baseline performances between real and sham trials were evaluated using Mann-Whitney test. A two-way repeated-measures analysis of variance (ANOVA) was run

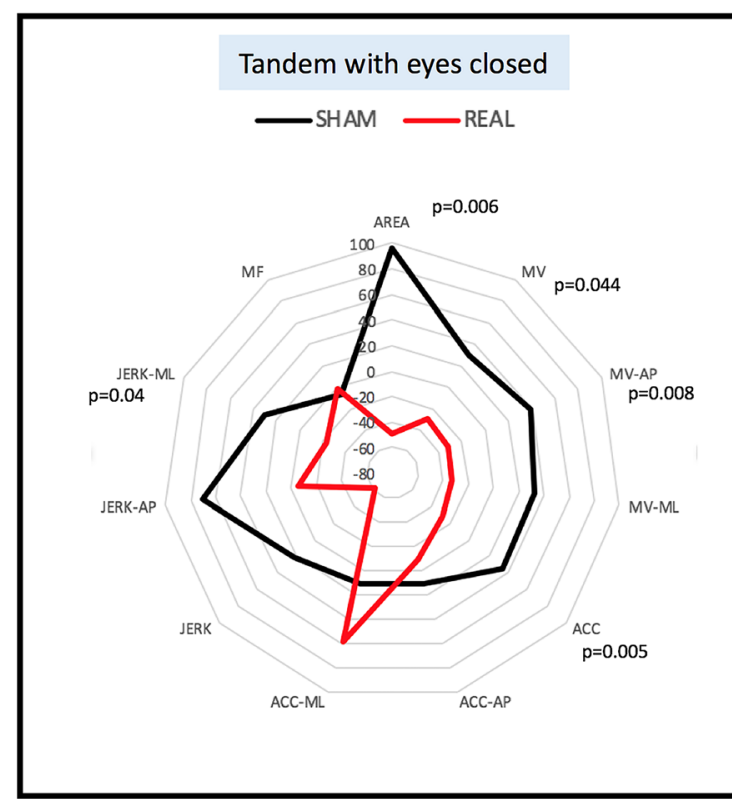

Fig. 1 Spider graphs showing the changes (in percentage) of static balance parameters from tandem stance and semitandem stance with eyes closed in patients with PSP, compared to values obtained before

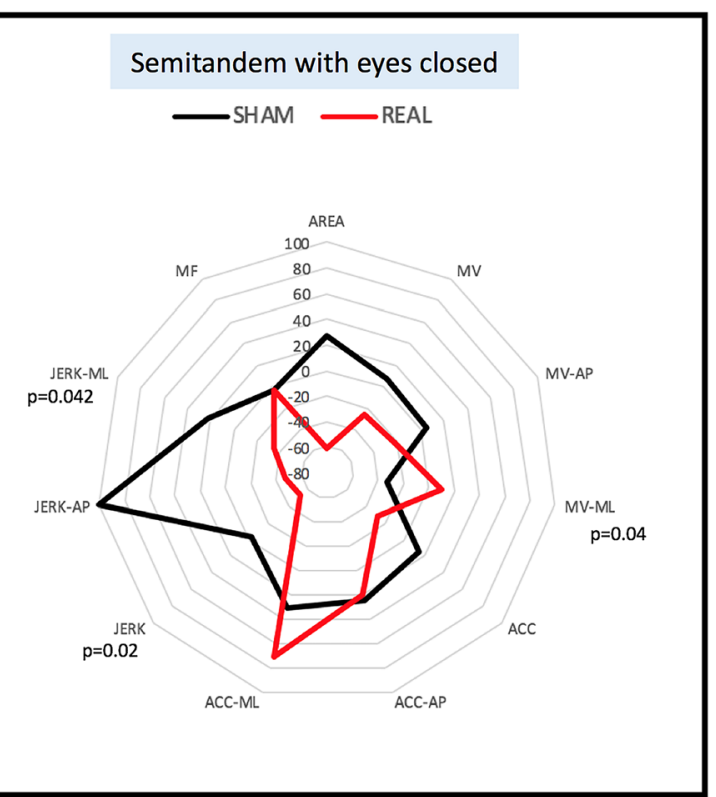

the sham versus real intervention. Significant differences are presented with $p$-values. ACC, acceleration; AP, anterior-posterior; MF, mean frequency; ML, medio-lateral; MV, mean velocity 
to determine the effect of the different treatments over time on assessment, adjusted for baseline values and the sequence of stimulation (real-sham vs sham-real). Significance was set to $p=0.05$, and SPSS software (version 21; SPSS, Inc., Chicago, IL) was used.

\section{Results}

\section{Study Cohort and rTMS}

Twenty PSP patients entered the study (mean age $74+4$ years, 13 males and 7 females). The mean disease duration was $3.8 \pm 1.2$ years, and the mean score on the PSP rating scale was $29 \pm 5$ points, with a mean levodopa equivalent dose of $417 \pm 89 \mathrm{mg} /$ day. All patients presented with postural instability, as reflected by specific PSPRS items and Tinetti scale. The rTMS protocol was well tolerated by all participants; side effects were neither reported nor observed during and after the stimulations.

There was no statistically significant association between type of stimulation and perception of patients $(p=0.89$, Fisher's exact test), suggesting that real rTMS could not be distinguished from sham stimulation.

\section{Dynamic Mobility and Mobile Health Technology- Instrumented Static Balance Assessment}

All patients were able to complete the 30-s semitandem/ tandem stance trials with eyes open, respectively. Sixteen participants completed the semitandem trial with eyes closed, and 14 the tandem trial with eyes closed. No differences in baseline performances in instrumented tests were detected for each task between real and sham stimulation. In both eyes closed conditions, the participants were able to stay longer without support after the real rTMS, compared to sham stimulation.

Table 1 Clinical and functional mobility parameters and static balance results in tandem and semitandem stances with eyes closed, before and after sham vs real cerebellar rTMS intervention performed in 20 patients with PSP

\begin{tabular}{|c|c|c|c|c|c|}
\hline Variable & Pre-SHAM & Post-SHAM & Pre-REAL & Post-REAL & $p$ \\
\hline \multicolumn{6}{|c|}{ Tandem eyes closed $(n=14)$} \\
\hline 30 -s task completed, $n$ & 9 & 7 & 8 & 7 & 0.8 \\
\hline Time, $\mathrm{s}$ & $21.1+12.2$ & $19.7+13.7$ & $20.9+11.8$ & $22.5+11.2$ & 0.046 \\
\hline Area, $\mathrm{mm}^{2}$ & $6.55 \mp 5.71$ & $12.8 \overline{1}+11.66$ & $25.4 \overline{2}+24.06$ & $12.79^{-}+15.26$ & 0.007 \\
\hline \multicolumn{6}{|l|}{ Velocity } \\
\hline $\mathrm{MV}, \mathrm{mm} / \mathrm{s}$ & $158.50+33.37$ & $206.89+102.57$ & $289.38+242.22$ & $202.87+85.49$ & 0.044 \\
\hline $\mathrm{MV}-\mathrm{AP}, \mathrm{mm} / \mathrm{s}$ & $20.04+\overline{1} 0.14$ & $27.76+\overline{1} 8.67$ & $35.97+\overline{2} 3.34$ & $24.42+\overline{1} 3.72$ & 0.009 \\
\hline MV-ML, mm/s & $77.99 \pm 65.36$ & $103.62 \pm 94.79$ & $134.9 \overline{7} \pm 199.56$ & $89.87 \pm 71.01$ & 0.299 \\
\hline \multicolumn{6}{|l|}{ Acceleration } \\
\hline $\mathrm{ACC}, \mathrm{mm} / \mathrm{s}^{2}$ & $27.01+12.16$ & $36.45+20.24$ & $48.80+29.57$ & $34.90+19.13$ & 0.005 \\
\hline ACC-AP, $\mathrm{mm} / \mathrm{s}^{2}$ & $62.10 \mp 41.89$ & $68.20 \mp 48.91$ & $84.01 \mp 72.00$ & $76.32 \overline{ \pm} 46.67$ & 0.544 \\
\hline ACC-ML, $\mathrm{mm} / \mathrm{s}^{2}$ & $0.97 \pm 0.80$ & $1.05 \pm 0.80$ & $1.94 \pm 2.84$ & $2.98 \pm 5.49$ & 0.575 \\
\hline \multicolumn{6}{|l|}{ Jerk } \\
\hline Jerk, $\mathrm{mm} / \mathrm{s}^{3}$ & $6.66 \pm 6.60$ & $8.14 \pm 6.36$ & $19.86 \pm 30.36$ & $7.42 \pm 5.33$ & 0.069 \\
\hline Jerk-AP, $\mathrm{mm} / \mathrm{s}^{3}$ & $1.02 \div 0.76$ & $1.69+2.06$ & $1.99+\overline{2} .70$ & $1.92 \div 2.97$ & 0.514 \\
\hline Jerk-ML, $\mathrm{mm} / \mathrm{s}^{3}$ & $17.5 \overline{3} \pm 8.25$ & $22.92 \pm 9.97$ & $31.68 \pm 20.66$ & $24.20 \pm 14.72$ & 0.040 \\
\hline \multicolumn{6}{|l|}{ Frequency } \\
\hline $\mathrm{MF}, \mathrm{Hz}$ & $1.34 \pm 0.36$ & $1.25 \pm 0.37$ & $1.29+0.51$ & $1.27+0.34$ & 0.647 \\
\hline \multicolumn{6}{|l|}{$\begin{array}{l}\text { Semitandem eyes } \\
\text { closed }(n=16)\end{array}$} \\
\hline 30-s task completed, $n$ & 9 & 9 & 9 & 9 & 1 \\
\hline Time, s & $26.7 \pm 7.0$ & $25.57+9.3$ & $26.5 \pm 12.5$ & $28.5 \pm 3.9$ & 0.046 \\
\hline Area, $\mathrm{mm}^{2}$ & $4.37 \mp 3.63$ & $5.57 \pm \overline{4} .31$ & $11.0 \overline{7} \pm 15.29$ & $4.35 \mp 3.01$ & 0.085 \\
\hline \multicolumn{6}{|l|}{ Velocity } \\
\hline $\mathrm{MV}, \mathrm{mm} / \mathrm{s}$ & $139.06 \pm 23.53$ & $149.12 \pm 38.57$ & 192.49 v 98.08 & $143.03 \pm 40.02$ & 0.083 \\
\hline MV-AP, mm/s & $16.76+5.28$ & $17.72+-10.04$ & $22.38+12.96$ & $17.26+\overline{7} .34$ & 0.167 \\
\hline MV-ML, mm/s & $106.5 \overline{1}+61.66$ & $72.27 \mp 63.99$ & $85.49 \mp 86.10$ & $94.17 \mp 75.60$ & 0.040 \\
\hline \multicolumn{6}{|l|}{ Acceleration } \\
\hline $\mathrm{ACC}, \mathrm{mm} / \mathrm{s}^{2}$ & $22.23 \pm 7.89$ & $25.28 \pm 10.88$ & $30.68 \pm 19.53$ & $22.19 \pm 8.42$ & 0.065 \\
\hline ACC-AP, $\mathrm{mm} / \mathrm{s}^{2}$ & $51.73 \pm 32.22$ & $64.44 \pm 38.06$ & $57.27 \mp 33.94$ & $68.90 \pm 69.31$ & 0.956 \\
\hline ACC-ML, $\mathrm{mm} / \mathrm{s}^{2}$ & $0.98 \pm 0.75$ & $1.31 \pm 1.71$ & $1.03 \pm 0.57$ & $1.70 \pm 1.00$ & 0.590 \\
\hline \multicolumn{6}{|l|}{ Jerk } \\
\hline Jerk, $\mathrm{mm} / \mathrm{s}^{3}$ & $3.98+2.47$ & $3.87 \pm 2.15$ & $7.29 \pm 4.61$ & $3.51 \pm 0.90$ & 0.021 \\
\hline Jerk-AP, $\mathrm{mm} / \mathrm{s}^{3}$ & $1.03 \pm 0.74$ & $2.01 \pm 3.49$ & $1.74 \pm 2.52$ & $0.92 \pm 1.25$ & 0.164 \\
\hline Jerk-ML, $\mathrm{mm} / \mathrm{s}^{3}$ & $14.08+7.14$ & $17.28+6.86$ & $20.56+15.27$ & $13.63+5.18$ & 0.042 \\
\hline \multicolumn{6}{|l|}{ Frequency } \\
\hline $\mathrm{MF}, \mathrm{Hz}$ & $1.26 \pm 0.33$ & $1.23 \pm 0.37$ & $137 \pm 0.35$ & $1.32 \pm 0.33$ & 0.845 \\
\hline
\end{tabular}

$A C C$ acceleration, $A P$ anterior-posterior, $M F$ mean frequency, $M L$ medio-lateral, $m m$ millimetre, $M V$ mean velocity, $R M S$ root mean square, $s$ seconds 
Moreover, in the tandem stance with eyes closed condition, the real intervention showed an improvement of the following parameters, compared to sham intervention: area, velocity, velocity in anterior-posterior direction, acceleration and jerk in the medio-lateral direction $(p<0.05$, Fig. 1$)$. In the semitandem stance with eyes closed condition, the real trial showed an increase of velocity in the medio-lateral direction and a decrease of global and medio-lateral jerkiness, compared to sham intervention (Table 1).

Results from the stance tasks with eyes open showed similar results, although less pronounced. Dynamic mobility assessment did not show differences in performance for real vs sham trial (Supplementary Table 1).

\section{Discussion}

Postural instability and falls are still important unmet therapeutic targets in PSP. The present trial, using a double-blind sham-controlled crossover design, suggests a beneficial effect of a single-session of cerebellar rTMS stimulation on measures of postural instability in PSP patients. Our results fit with the observation of alterations of the cerebellum in PSP [2, 3, 5, $6]$, and with preliminary evidence coming from pilot studies.

The first published rTMS open-label trial in PSP using cerebellar theta burst stimulation in 10 patients showed an improvement of functional connectivity between cerebellum and motor cortex assessed by neurophysiological measures (i.e. CBI) and functional MRI [7]. However, the trial could not exclude a placebo effect due to the open-label design. Moreover, the authors could not demonstrate a relevant clinical effect. This aspect was recently addressed by a shamcontrolled rTMS case study performed for 10 days in two PSP patients, showing an improvement in CBI and posturography in the real intervention, although not significant due probably to an unexpected placebo effect in one patient [8].

The present trial adds novel insights into this concept on multiple levels. First, it provides information about a reasonably large cohort of early stages PSP and uses a high-quality design. Second, it considered several assessment strategies, including novel mobile health technology, to assess even subtle but potentially clinically relevant parameters. At variance with previous studies applying multiple sessions, a single theta burst stimulation showed a surprisingly clear effect on accelerometer-derived measures of static balance, a feature that is regularly affected in PSP and leads to severe impairment in daily activities and quality of life. The real intervention showed an effect particularly in the medio-lateral direction of static balance (see, e.g. Table 1 and Supplementary Table 1). This is of interest, as MacKinnon and colleagues [13] and Mitchell and colleagues [14] found that mediolateral parameters of static balance reflect predominantly axial and antero-posterior parameters predominantly distal compensatory movements. In our assessments, PSP patients were able to extend the time they could perform the tasks after real stimulation, making us optimistic for the clinical translation of rTMS stimulation. Third, the improvement in static balance, as observed with mobile health technology, was not reflected by any of the clinical and mobility performance test included in the assessment battery. This result highlights the need for inclusion of such technology in these types of trials, as conventional assessment methods may be too roughly scaled to detect relatively subtle changes. Fourth, the effects of rTMS on static balance parameters were particularly evident in the more challenging tasks. This aspect argues for the usefulness of challenging paradigms to be included in assessment panels of clinical trials together with neuronavigation and more complex measures (including unsupervised assessments [15]). The response of a single-session rTMS could also identify those patients who might benefit most of longer rTMS trials to be performed in the future.

In conclusion, this is the first study showing a relevant effect of a short cerebellar rTMS intervention on static balance in PSP patients, supporting the rationale for longer stimulation protocols.

Supplementary Information The online version contains supplementary material available at https://doi.org/10.1007/s12311-021-01239-6.

Acknowledgements The authors thank the study participants and the physiotherapists involved in the study. Without their invaluable contribution, this study would not have been possible.

Funding Open Access funding provided by Università degli Studi di Brescia within the CRUI-CARE Agreement.

\section{Declarations}

Conflict of Interest The authors declare no competing interests.

Open Access This article is licensed under a Creative Commons Attribution 4.0 International License, which permits use, sharing, adaptation, distribution and reproduction in any medium or format, as long as you give appropriate credit to the original author(s) and the source, provide a link to the Creative Commons licence, and indicate if changes were made. The images or other third party material in this article are included in the article's Creative Commons licence, unless indicated otherwise in a credit line to the material. If material is not included in the article's Creative Commons licence and your intended use is not permitted by statutory regulation or exceeds the permitted use, you will need to obtain permission directly from the copyright holder. To view a copy of this licence, visit http://creativecommons.org/licenses/by/4.0/.

\section{References}

1. Höglinger GU, Respondek G, Stamelou M, Kurz C, Josephs KA, Lang AE, et al. Clinical diagnosis of progressive supranuclear 
palsy: the movement disorder society criteria. Mov Disord. 2017;32:853-64. https://doi.org/10.1002/mds.26987.

2. Gellersen HM, Guo CC, O'callaghan C, Tan RH, Sami S, Hornberger M. Cerebellar atrophy in neurodegeneration - a metaanalysis. J Neurol Neurosurg Psychiatry. 2017;88(9):780-8.

3. Dickson DW, Ahmed Z, Algom AA, Tsuboi Y, Josephs KA. Neuropathology of variants of progressive supranuclear palsy. Curr Opin Neurol. 2010;23:394-400. https://doi.org/10.1097/ WCO.0b013e32833be924.

4. Tramontano M, Grasso MG, Soldi S, Casula EP, Bonnì S. Mastrogiacomo S, et al. Cerebellar intermittent theta-burst stimulation combined with vestibular rehabilitation improves gait and balance in patients with multiple sclerosis: a preliminary double-blind randomized controlled trial cerebellum. 2020 Dec;19(6):897-901. https://doi.org/10.1007/s12311-020-01166-y.

5. Shirota Y, Hamada M, Hanajima R, Terao Y, Matsumoto H, Ohminami S, et al. Cerebellar dysfunction in progressive supranuclear palsy: a transcranial magnetic stimulation study. Mov Disord . 2010; 25:2413-2419. doi:https://doi.org/10.1002/ mds.23298.

6. Benussi A, Dell'Era V, Cantoni V, Turrone R, Pilotto A, Alberici $A$, et al. Stimulation over the cerebellum with a regular figure-ofeight coil induces reduced motor cortex inhibition in patients with progressive supranuclear palsy. Brain Stimul. 2019;12(5):1290-7. https://doi.org/10.1016/j.brs.2019.05.017.

7. Brusa L, Ponzo V, Mastropasqua C, Picazio S, Bonnì S, Di F, et al. Brain stimulation theta burst stimulation modulates cerebellarcortical connectivity in patients with progressive supranuclear palsy. Brain Stimul. 2014;7:29-35. https://doi.org/10.1016/j.brs.2013. 07.003 .

8. Dale ML, DeVries WH, Mancini M, George MS. Cerebellar rTMS for motor control in progressive supranuclear palsy. Brain Stimul. 2019;12(6):1588-91 http://www.ncbi.nlm.nih.gov/pubmed/ 31378601 .
9. Golbe LI, Ohman-Strickland PA. A clinical rating scale for progressive supranuclear palsy. Brain. 2007;130:1552-65 http://www. ncbi.nlm.nih.gov/pubmed/17405767.

10. Pilotto A, Gazzina S, Benussi A, Manes M, Dell'Era V, Cristillo V, et al. Mild cognitive impairment and progression to dementia in progressive supranuclear palsy. Neurodegener Dis. 2017;7(6): 286-91. https://doi.org/10.1159/000479110.

11. Geritz J, Maetzold S, Steffen M, Pilotto A, Corrà MF, Moscovich $\mathrm{M}$, et al. Motor, cognitive and mobility deficits in 1000 geriatric patients: protocol of a quantitative observational study before and after routine clinical geriatric treatment - the ComOn-study. BMC Geriatr. 2020 Feb 6;20(1):45. https://doi.org/10.1186/s12877-0201445-z.

12. Mancini M, Salarian A, Carlson-Kuhta P, Zampieri C, Laurie King, Lorenzo Chiari et al. ISway: a sensitive, valid and reliable measure of postural control Neuroeng Rehabil. 2012 Aug 22;9:59. doi: https://doi.org/10.1186/1743-0003-9-59.

13. MacKinnon CD, Winter DA. Control of whole body balance in the frontal plane during human walking. J Biomech. 1993;26:633-44. https://doi.org/10.1016/0021-9290(93)90027-C.

14. Mitchell SL, Collin JJ, De Luca CJ, Burrows A, Lipsitz LA. Openloop and closed-loop postural control mechanisms in Parkinson's disease: increased mediolateral activity during quiet standing. Neurosci Lett. 1995 Sep;197(2):133-6. https://doi.org/10.1016/ 0304-3940(95)11924-1.

15. Warmerdam E, Hausdorff JM, Atrsaei A, Zhou Y, Mirelman A, Aminian $\mathrm{K}$, et al. Long-term unsupervised mobility assessment in movement disorders. Lancet Neurol. 2020;4422:1-9. https://doi. org/10.1016/S1474-4422(19)30397-7.

Publisher's Note Springer Nature remains neutral with regard to jurisdictional claims in published maps and institutional affiliations. 